\title{
Análisis de los factores de reabsorción de líquido de irrigación durante la RTU de próstata
}

\author{
O. Regojo Zapata, A. Elizalde Benito, J. Navarro Gil, I. Hijazo Conejos, J.M. Sánchez Zalabardo, \\ J.G. Valdivia Uría
}

Servicio de Urología. Hospital Clínico Universitario Lozano Blesa. Zaragoza.

Actas Urol Esp 2005; 29 (2): 174-178

\section{RESUMEN}

ANÁLISIS DE LOS FACTORES DE REABSORCIÓN DE LÍQUIDO DE IRRIGACIÓN DURANTE LA RTU DE PRÓSTATA

Objetivos: En este estudio fue nuestra intención evaluar la relación entre la reabsorción de líquido de irrigación y tres variables: tiempo de la intervención, volumen de solución de glicina empleado y peso de los fragmentos, durante la RTU de próstata realizada a baja presión hidráulica.

Material y método: Estudiamos 74 pacientes a los que se realizó RTU de próstata con derivación suprapúbica con vaina de Amplatz $30 \mathrm{ch}$. Se monitorizó el etanol en aire expirado cada 15 minutos durante la intervención. Así mismo se anotaron el tiempo de la intervención, el volumen de glicina utilizado y el peso de los fragmentos extraídos. Estadísticamente se utilizó el Test de Anova para comparación de medias.

Resultados: Un 13,6 \% de los pacientes absorbieron líquido de irrigación en alguna cantidad. El rango de absorción es de 100 cc hasta 2.000 cc. No se encontró una diferencia estadísticamente significativa entre las medias del tiempo de resección, del volumen de glicina y del peso de los fragmentos entre el grupo de pacientes que había reabsorbido líquido de irrigación y los que no reabsorbieron.

Conclusiones: Nuestro estudio pone de manifiesto que el tiempo operatorio, el volumen de líquido de irrigación y el peso de los fragmentos resecados no influyen en la reabsorción de líquido de irrigación cuando se realiza una RTU a baja presión hidráulica.

Palabras clave: Resección transuretral de próstata. Síndrome RTU. Monitorización etanol. Hiponatremia. Amplatz suprapúbico.

\section{ABSTRACT}

\section{ANALYSIS OF THE ABSORCION'S FACTORS OF IRRIGATING FLUID DURING TRANSURETHRAL} RESECTION OF THE PROSTATE

Objetives: In this study it was our intention to evaluate the relation between the reabsorption of irrigating fluid and three variables: time of the intervention, volume of solution of glicina employee and weight of the fragments, during the RTU of prostate fulfilled to low hydraulic pressure.

Material and method: We Study 74 patients that RTU of prostate was performed with suprapúbica derivation with Amplatz's pod $30 \mathrm{ch}$. The ethanol was monitored in expired air every 15 minutes during the intervention. Likewise we annotated the time of the intervention, the volume of glicina used and the weight of the fragments extracted. Statistically Anova's text was in use for comparison of averages.

Results: $13,6 \%$ of the patients absorbed irrigating fluid in some quantity. The range of absorption belongs to $100 \mathrm{cc}$ until $2.000 \mathrm{cc}$. We did not find a statistically significant difference in the averages of time of resection, volume of glicina and weight of the fragments between the group of patients that had absortion of irrigating fluid and they that didn't had.

Conclusions: Our data show that the operative time, the volume of irrigating fluid and the weight of the resected fragments do not influence the reabsorption of liquid of irrigation when a RTU is realized to low hydraulic pressure.

Keywords: Transuretral resection of prostate. TUR Syndrome. Monitoring ethanol. Hiponatremia. Suprapubic amplatz. 
$\mathrm{L}^{2}$ a Hiperplasia benigna de próstata (HBP.) supone hoy, en nuestra sociedad occidental, un problema sanitario de primer orden ${ }^{1}$. El tratamiento farmacológico representa en la actualidad el primer paso terapéutico, en los casos de indicación relativa de cirugía y su impacto ha hecho disminuir algo el número de Resecciones transuretrales de próstata (RTUs) ${ }^{2}$. Aun con todo, la cirugía sigue siendo el único tratamiento curativo de la enfermedad.

Dentro de la cirugía, en los últimos años han proliferado distintas formas de abordar el problema. De esos tratamientos alternativos los únicos que se aceptan hoy en día por sus beneficios demostrados son: cirugía abierta, láser Holmium y RTU. convencional. Este último es el utilizado por el $90 \%$ de los urólogos ${ }^{2}$.

El estudio de Mebust et al. ${ }^{3}$ donde se valoraron las complicaciones postoperatorias presentadas tras la RTU de próstata en 13 centros participantes, abarca a 3.885 pacientes y habla de un claro descenso de mortalidad global de esta técnica quirúrgica, que hoy se sitúa en el 0,2\%; sin embargo recordaba un hecho que no ha cambiado en los últimos años: la elevada tasa de morbilidad postoperatoria: 18\%. Esto no es extraño si tenemos en cuenta que el 77\% de estos pacientes tiene una historia clínica patológica importante. Este estudio sitúa como primera causa de morbilidad intra operatoria el sangrado que requiere transfusión, que ocurrió en el 2,5\% de las RTU La segunda causa de morbilidad intra operatoria, con un 2\% de aparición es el síndrome de la RTU.

Hasta el momento han aparecido distintos tipos de soluciones no hemolíticas: Glicina al $1,2 \%$, glicina al 1,5\%, sorbitol 3,5\%, manitol 5\%, cytol, glucosa $2,5 \%$ y urea $1 \%{ }^{6}$. Todas ellas con enormes ventajas sobre el agua, que se utilizaba antes. A pesar de la gran variedad de soluciones la que se ha popularizado más es la glicina al $1,5 \%$, que da excelente resultados. La glicina reúne las mejores condiciones al ser un aminoácido sintetizado por el cuerpo humano, y hace que la solución sea isoeléctrica, con lo que consigue no difundir la corriente eléctrica monopolar del resector y es mínimamente hipotónica con lo que se evita la hemólisis cuando la solución pase al torrente sanguíneo ${ }^{4-6}$.
Las rutas de absorción del líquido de irrigación son fundamentalmente dos: intravascular y extravascular ${ }^{7}$. La intravascular se produce a través de los senos venosos prostáticos abiertos, a expensas de la presión intravesical. La extravascular se produce a través de las perforaciones de la cápsula prostática, produciéndose la acumulación de líquido lavador en tejido conectivo perivesical.

Hoy este síndrome se describe como una hiponatremia y una clínica neurológica que se produce por la acción de la glicina a nivel de sistema nervioso central. La sintomatología ha sido muy bien descrita y supone: Alteraciones neurológicas (92\% de los casos), Alteraciones cardiovasculares (54\%), Alteraciones visuales $(42 \%)^{10}$ y alteraciones digestivas $(25 \%)^{8}$ En un estudio de Radal et $\mathrm{al}^{9}$ basado en casos severos (Hiponatremias $113+-6 \mathrm{mmol} / 1)$ constató una mortalidad del 25\% en dicho grupo.

El estudio de la absorción de líquido de irrigación en la RTU no es algo novedoso. Estas experiencias comenzaron en la década de los 60 con la determinación del peso del paciente antes y después de la intervención ${ }^{11}$ Años más tarde se intentó la aproximación a la cantidad de líquido absorbido con distintos marcadores: salicilato sódico ${ }^{12}$, albúmina marcada con radioisótopo (I $131)^{13}$, etc.

En la década de los 80 son Hulten y Hahn los que revolucionaron estos estudios introduciendo un nuevo marcador, fácil de utilizar e inocuo, el Etanol $^{14}$ Según la ley de Henry, la proporción de alcohol en la sangre y en el aliento se relaciona entre sí de forma muy precisa, de forma que podemos conocer por medio de un alcoholímetro de aire espirado la cantidad exacta de alcohol en sangre $^{15}$.

A partir de estos conocimientos se van multiplicando la literatura urológica y anestésica en este campo. Hahn ${ }^{16}$ demuestra que la concentración más rentable de Etanol para esta práctica es la solución al 1\%. Gehring ${ }^{17}$ estudia la interferencia del Etanol y los gases anestésicos en la RTU realizada bajo anestesia general ${ }^{18}$. Aragona ${ }^{19}$ estudia la reabsorción de líquido con el método de Etanol en RTU a baja presión. Otros estudios han demostrado la utilidad del etanol como buen marcador de reabsorción ${ }^{20-21}$. 
Los estudios que hoy se realizan van encaminados a describir las tasas de reabsorción, a descubrir los principales factores de la absorción de líquido de irrigación y del síndrome post $\mathrm{RTU}^{22-31}$.

Los objetivos de este estudio fueron:

1. Estudio descriptivo de las tasas de absorción de líquido de irrigación e incidencia del "Síndrome RTUP" en nuestro Servicio.

2. Determinar, si existe, una relación estadísticamente significativa entre la reabsorción de líquido de irrigación y tres variables: peso de los fragmentos, volumen del líquido de irrigación y tiempo de la intervención.

Proponemos como hipótesis del estudio: La reabsorción de líquido de irrigación durante la RTU de próstata es mayor, cuanto mayor es el peso de los fragmentos resecados, el tiempo operatorio y el volumen de glicina consumido.

\section{MATERIAL Y MÉTODO}

El estudio se realiza en pacientes con hiperplasia benigna de próstata (HBP) a los que se les indica RTU como tratamiento definitivo de su patología. Dentro de los criterios de inclusión están que el paciente no haya sido sometido a RTU de próstata previa y que el adenoma sea menor de $100 \mathrm{cc}$ medido por ecografía. Utilizamos en todos los casos derivación suprapúbica con vaina de amplatz de $30 \mathrm{ch}$ para conseguir baja presión hidráulica en celda prostática durante toda la intervención. Se excluye los pacientes que requieren anestesia general para la intervención, por la imposibilidad de registrar las tasas de etanol en aire espirado. Del mismo modo, excluimos a los pacientes con incapacidad mental o auditiva importante, que les haga imposible la comprensión de la técnica de soplado en el alcoholímetro. No se excluye ningún paciente por el riesgo anestésico ya que esto nos podría sesgar el estudio. Se requirió de los pacientes el consentimiento expreso para participar en este estudio. En cada paciente se recogen los datos demográficos: edad, estado, domicilio, etc. Se extraen asimismo los datos de más relevantes de su historia clínica: Antecedentes patológicos, peso, talla e historia clínica urológica. Durante la intervención se rellena la ficha de datos intra operatorios que recoge: Etanol en aire espirado preoperatorio, a los 15 min del comienzo de la resección, a los 30,
45, 60, 75,90 y $105 \mathrm{~min}$. Se lleva acabo el pesaje de los fragmentos, la contabilización de los litros de líquido de irrigación empleados y anotación del tiempo de resección.

En otro apartado de la ficha se rellenan las incidencias intra operatorias:

1. No incidencias.

2. Perforación de la cápsula.

3. Apertura de senos venosos.

4. Salida de la derivación suprapúbica.

En otra ficha aparte se recogen la aparición de síntomas post operatorios:

1. No sintomas.

2. Nauseas y vómitos.

3. Hipotensión.

4. Mareos.

5. Dolor pectoral.

6. Ceguera transitoria.

7. Obnubilación.

Deseamos saber si la diferencia de medias en cada variable dependiente es significativa y consideramos que seria objetiva la diferencia de 10 puntos (g de próstata, tiempo de resección y volumen de líquido de irrigación). Por estudios previos sabemos que la desviación típica de los items estudiados está entre 15 y 25 puntos (utilizaremos 20 como media). Aceptamos un riesgo de 0.05 y deseamos un poder estadístico de $90 \%$ para detectar diferencias si es que existen.

$$
n=\frac{2\left(Z_{a}+Z_{a}\right)^{2 *} S^{2}}{d^{2}}
$$

$$
\mathrm{n}=2(1.65+1.282)^{2} * 20^{2} \quad / \quad 10^{2}=68,7
$$

Precisamos unos 68 pacientes en el estudio. Se prevé un $8 \%$ de posibles pérdidas de información durante la ejecución del estudio, el tamaño muestral debe recalcularse según la siguiente expresión: $\mathrm{n}^{\prime}=\mathrm{n} / 1-\mathrm{L}=68 / 1-0,08=73,9$.

La técnica que se utiliza para comparar medias se llama análisis de la varianza, el objeto es la comparación entre medias, en nuestro caso debemos comparar las medias de las variables estadísticas cuando ha habido reabsorción y cuando no. Los datos se incluyen en una base de datos y el análisis estadístico se realiza con Stat view.

\section{RESULTADOS}

Un total de 10 pacientes (13,6 \%) absorbieron líquido de irrigación en alguna cantidad (Fig. 1). 


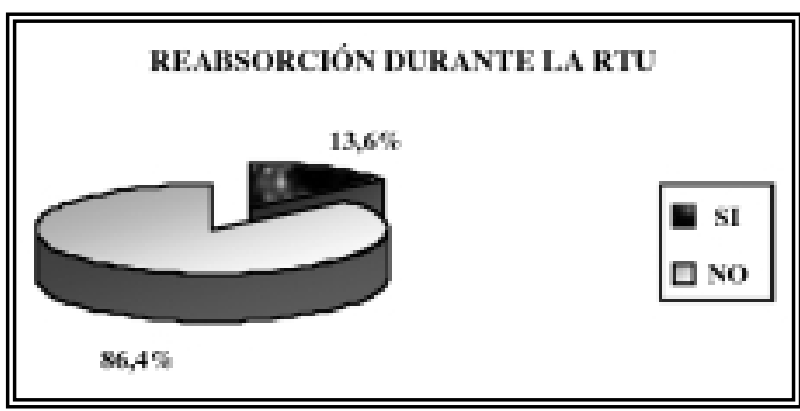

FIGURA 1. Proporción de reabsorción en las RTU realizadas.

En la literatura no se encuentra una tasa de reabsorción tan baja. Posiblemente se deba a la utilización de la técnica de RTU a baja presión hidráulica (Fig 2).

La media de volumen absorbido en estos pacientes fue de $518 \mathrm{ml}$ (Rango 100-2.000 ml). En 9 pacientes se produjo una reabsorción menor de $800 \mathrm{ml}$ que no requirió ninguna medida durante la intervención. En 1 caso se produjo una perforación accidental al principio de la resección, con reabsorción masiva de $2000 \mathrm{ml}$, que requirió terminar la RTU (Fig. 3).

Ninguno de estos 10 pacientes presentó síntomas compatibles con el sindrome post-RTU.

La media de tiempo de intervención de los pacientes que reabsorbieron fue de 59,5 min. Mientras que los pacientes que no reabsorbieron tenían una media de tiempo de 55,3 min. La media de volumen de líquido de irrigación de los

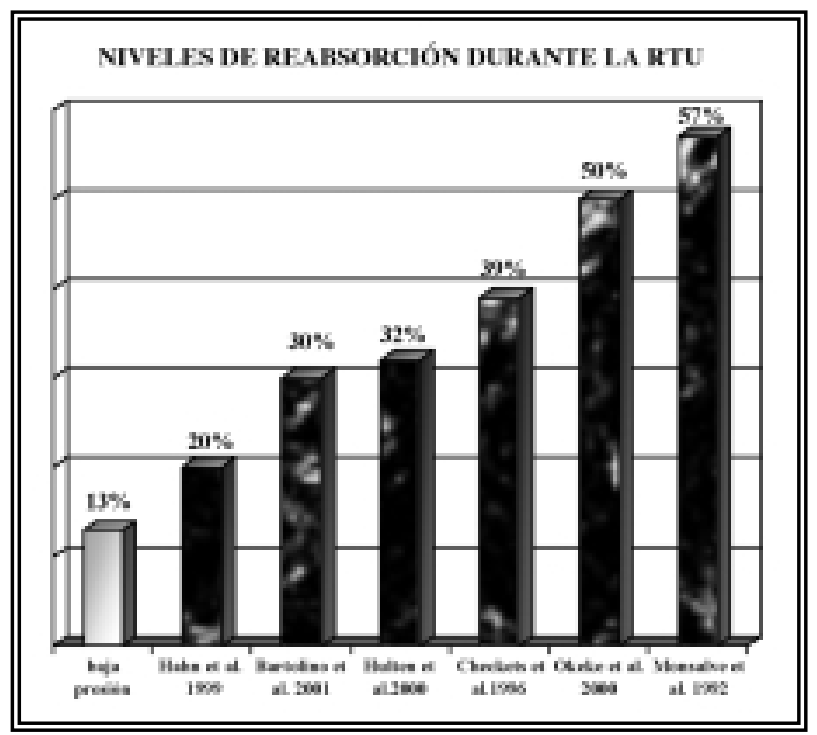

FIGURA 2. Proporción de reabsorción en varias series de la literatura.

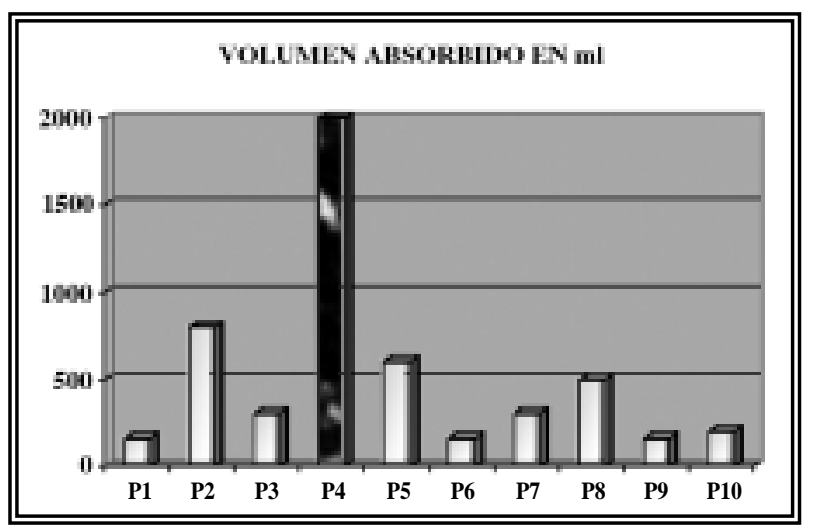

FIGURA 3. Volumen de liquido de irrigación reabsorbido en cada una de los pacientes en los que se detectó etanol en aire espirado.

pacientes que reabsorbieron fue de $30,7 \mathrm{~L}$. Mientras que los pacientes que no reabsorbieron tenían una media de volumen de 28,7 L. La media de peso de los fragmentos de próstata resecada de los pacientes que reabsorbieron fue de $37,7 \mathrm{~g}$. Mientras que los pacientes que no reabsorbieron tenían una media de peso de $38,7 \mathrm{~g}$ (Tabla 1 ).

No se encontró una diferencia estadísticamente significativa entre las medias de las tres variables estudiadas en el análisis de la varianza. (La diferencia se debía al azar). (Fig. 4).

\section{Tabla 1}

Media y desviación estándar de las tres variables en los grupos de reabsorción y no-reabsorción

\begin{tabular}{lccccccc}
\hline & \multicolumn{2}{c}{$\begin{array}{c}\text { Tiempo de } \\
\text { reacción }\end{array}$} & \multicolumn{2}{c}{$\begin{array}{c}\text { Volumen } \\
\text { glicina }\end{array}$} & \multicolumn{2}{c}{$\begin{array}{c}\text { Peso } \\
\text { fragmentos }\end{array}$} \\
& Media & D.E. & Media & D.E. & Media & D.E. \\
Reabsorción & 59,5 & 25,7 & 30,7 & 12,2 & 37,7 & 24,5 \\
No reabsorción & 55,3 & 23,3 & 28,7 & 14,0 & 38,7 & 24,6 \\
\hline
\end{tabular}

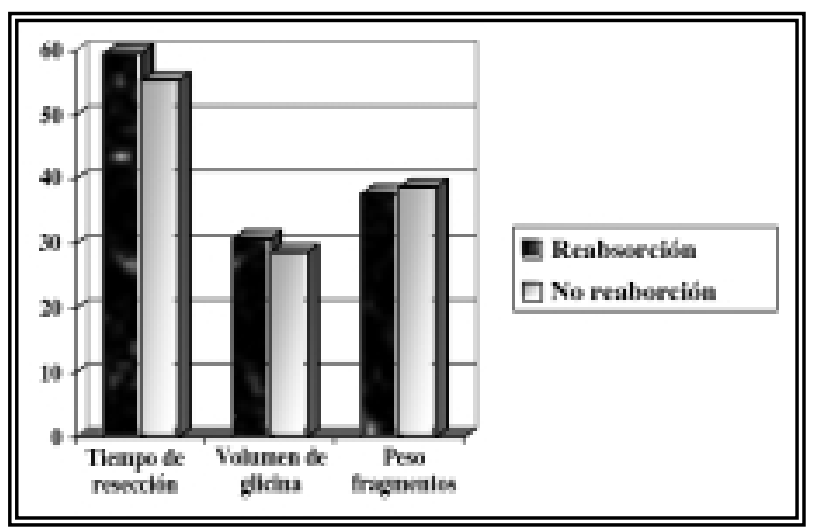

FIGURA 4. Comparación de medias de las tres variables. 


\section{CONCLUSIONES}

1. Debemos rechazar la hipótesis de trabajo del estudio y concluir que el tiempo operatorio, el volumen de líquido de irrigación y el peso de los fragmentos resecados no influyen en la reabsorción de líquido de irrigación cuando se realiza una RTU a baja presión hidráulica.

2. El porcentaje bajo de reabsorción con relación a otras publicaciones, nos hace suponer que la técnica de RTU a baja presión es un factor protector de la reabsorción y por consiguiente del sindrome post RTU.

3. Los estudios que se llevan a cabo en este proyecto de investigación se dirigirán ahora a encontrar una variable que se relacione con la reabsorción de líquido de irrigación. Asímismo se está estudiando la posible variación de la absorción del líquido de irrigación con distintas técnicas de RTU de próstata.

\section{REFERENCIAS}

1. De la Rossete J, Perachino M, Thomas D, et al. Guidelinesen on bening prostatic hiperplasia. In EUA Guidelines 2001;1-63.

2. Vicente Rodríguez J. Tratamiento de la HBP Realidades presentes y perspectivas futuras. Actas urológicas Españolas 2002;481-490.

3. Mebust WK, Holtgrewe HL, Cockett AT, Peters PC. Transurethral prostatectomy: immediate and postoperative complications. a cooperative study of 13 participating institutions evaluating 3,885 patients. J Urol 2002 Feb; 167(2 Pt 2):999-1003.

4. Creevy CD. Hemolytic reactions during transurethral prostatic resection. J Urol 1947;58:125.

5. Emmett JL, James H, Gilbaugh JR, Mclean P. Fluid absorption during transurethral resection: comparison of mortality and morbidity after irrigation with water and non-hemolytic solutions. J Urol 1969;101:884-889.

6. Vidal A, Angulo JC. Síndrome de reabsorción postresección transuretral de próstata. Rev Urol 2002;3(2):73-77.

7. Olsson J, Nilsson A, Hahn RG. Syntoms of the transurethral resection symdrome using glycine as the irrigant. J Urol 1995;154:123128.

8. Clemente Ramos LM, Ramasco Rueda F, Platas Sancho A, Archilla Esteban J, Romero Cajigal I, Corbacho Fabregat C, Allona Almagro A. Síndrome de reabsorción post-resección transuretral (RTU) de próstata: revisión de aspectos fisiopatológicos, diagnósticos y terapéuticos. Actas Urol Esp 2001; 25(1):14-31.

9. Radal M, Bera AP, Leisner C, Haillot O, Autret-Leca E. Adverse effects of glycolic irrigation solutions. Therapie 1999;54(2):233-236.

10. Wang JM, Creel DJ, Wong KC. Transurethral resection of the prostate, serum glycine levels, and ocular evoked potentials. Anesthesiology. 1989;70(1):36-41.

11. Oester A, Madsen PO. Determination of absorption of irrigating fluid during transurethral resection of the prostate by means of radioisotopes. J Urol. 1969;102(6):714-719.

12. Landsteiner EK, Finch CA. Hemoglobinemia accompanying tranurethral resection of the prostate. New Engl. J Med 1947;237:310.

13. Griffin M, Dubson L, Weaver JC. Volumen of irrigation fluid transfer during transurethral prostatectomy, studied with radioisotopes. J Urol. 1955. 74: 646.
14. Hulten JO, Jorfeldt LS, Wictorsson YM. Monitoring fluid absorption during TURP by marking the irrigating solution with ethanol. Scand J Urol Nephrol 1986;20(4):245-51.

15. Manual de análisis de alcohol en aire espirado. Dräger.

16. Hulten JO, Hahn RG. Monitoring irrigating fluid absorption during transurethral resection of the prostate (TURP); a comparison between 1 and $2 \%$ ethanol as a tracer. Scand J Urol Nephrol 1989; 23(2): 103-8.

17. Gehring H, Nahm W, Klotz KF, Knipper A, Zimmermann K, Baerwald J, Schmucker P. Measurement of expired alcohol concentrations with a new electrochemical sensor. A model investigation to deter mine interference with volatile anesthetics and clinical application. Anaesthesist 1996;45(2): 154-162.

18. Gehring $\mathrm{H}$, et al. Measurement of breath ethanol concentration in patients with general. Anesthesiology 1997;87: No 3A. A406.

19. Aragona F, Panza N, Mangano A, Castellucci E, Capizzi A. No evidence of fluid absorption during continuous low-pressure transurethral resection of the prostate: assessment by measuring expiratory breath ethanol concentrations. Eur Urol 1999;36(2):103106.

20. Gehring H, Hornberger C, Dibbelt L, Dorges V, Eichenauer R, Schmucker P. Detecting and quantifying absorbed irrigation fluid by measuring mannitol and sorbitol concentrations in serum samples, and by ethanol monitoring. BJU Int 2002;89(3):202-207

21. Hahn RG. Ethanol monitoring of irrigating fluid absorption in transurthral protatic surgery. Anesthesiology 1988;V68. No6. 867873.

22. Expired breath ethanol measurement to calculate irrigating fluid absorption during transurethral resection of the prostate: experience in a district general hospital. Br J Urol 1996;77(2):198-202.

23. Hahn RG, Ekengren J. Absorption of irrigating fluid and height of fluid bag during transurethral resection of the prostate. $\mathrm{Br} \mathrm{J}$ Urol 1993;72(1):80-83.

24. Hahn RG. Early detection of the TUR syndrome by marking the irrigating fluid with 1\% ethanol. Acta Anaesthesiol Scand 1989;33(2): 146-151.

25. Bartoloni A, Gottin L, Ficarra V, Capotosto C, Malossini G, Tallarigo C, Finco G. The TURP syndrome: importance of expiratory ethanol measurement and high serum levels of glycine. Arch Esp Urol 2001; 54(5):480-487

26. Hulten JO, Tran VT, Pettersson G. The control of haemolysis during transurethral resection of the prostate when water is used for irrigation: monitoring absorption by the ethanol method. BJU Int 2000; 86(9):989-992.

27. Monsalve $\mathrm{C}$ et al. Monitorización de la absorción de la solución de irrigación en la resección transuretral de próstata, utilizando etanol como marcador. Rev. Esp. Anestesiol 1992;39(Sup 1):118.

28. Okeke AA, Lodge R, Hinchliffe A, Walker A, Dickerson D, Gillatt DA. Ethanol-glycine irrigating fluid for transurethral resection of the prostate in practice. BJU Int;2000:86(1):43-46.

29. Hahn RG. Irrigating fluid in endoscopic surgery. Br J Urol 1997;79: 669-680.

30. Hahn RG. Fluid and electrolyte dynamics during development of the TURP syndrome. Br J Urol 1990;66(1):79-84.

31. Istre O, Bjoennes J, Naess R, Hornbaek K, Forman A. Postoperative cerebral oedema after transcervical endometrial resection and uterine irrigation with 1.5\% glycine. Lancet;1994:344(8931):1187-1189.

\section{Dr. O. Regojo Zapata \\ C/ Lastanosa 17, $1^{\text {o }}$ \\ 50010 Zaragoza}

E-mail: oscarregojo@terra.es

(Trabajo recibido el 2 septiembre de 2004) 